\title{
Some fixed point theorems for Meir-Keeler condensing operators with applications to integral equations
}

\author{
A. Alotaibi \\ M. Mursaleen \\ S.A. Mohiuddine
}

\begin{abstract}
In this paper, we prove some tripled fixed point theorems for Meir-Keleer condensing operator in a Banach space by using $L$-functions. We apply these results to establish the existence of solutions for a system of functional integral equations of Volterra type.
\end{abstract}

\section{Introduction and preliminaries}

The degree of noncompactness of a set is measured by means of functions called measures of noncompactness. The first measure of noncompactness, the function $\alpha$, was defined and studied by Kuratowski [24] in 1930. Darbo [16] used this measure to generalize both the Schauder's fixed point theorem and the Banach's contraction principle for so called condensing operators. The Hausdorff MNC $\chi$ was introduced by Goldenstein, Gohberg and Markus [20] in 1957 and later studied by Goldenstein and Markus [21]. Another measure of noncompactness $\beta$ was introduced by Istrățescu [22] in 1972. Measures of noncompactness are very useful tools which are widely used in fixed point theory, differential equations, functional equations, integral and integro-differential equations, and optimization etc. [11]. In recent years measures of noncompactness have also been used

Received by the editors in November 2014 - In revised form in February 2015.

Communicated by E. Colebunders.

2010 Mathematics Subject Classification : 47H09, 47H10.

Key words and phrases : Measure of noncompactness, Darbo's fixed point theorem, triple fixed point, Meir-Keeler condensing operators, Volterra integral equation. 
in defining geometric properties of Banach spaces as well as in characterizing compact operators between sequence spaces, e.g. [30].

For a bounded subset $Q$ of a metric space $E$, the Kuratowski measure of noncompactness ( $\alpha$-measure or set measure of noncompactness) of $Q$ is defined by

$$
\alpha(Q)=\inf \left\{\epsilon>0: Q \subset \bigcup_{i=1}^{n} S_{i}: S_{i} \subset E, \operatorname{diam}\left(S_{i}\right)<\epsilon(i=1, \ldots, n) ; n \in \mathbb{N}\right\} .
$$

Another measure of noncompactness is the Hausdorff measure of noncompactness ( $\chi$-measure or ball measure of noncompactness), which is more applicable in many cases. It is defined by the formula

$$
\chi(Q)=\inf \{\varepsilon>0: Q \text { has a finite } \varepsilon \text {-net in } E\} .
$$

The two measures $\chi$ and $\alpha$ share many properties $[7,10]$. Here, we recall some basic facts concerning measures of noncompactness (c.f. [10]). We denote the set of real numbers by $\mathbb{R}$ and put $\mathbb{R}_{+}=[0, \hat{A}+\infty)$. Let $(E,\|\|$.$) be a Banach$ space. The symbol $\bar{Q}, \operatorname{Conv} Q$ will denote the closure and closed convex hull of a subset $Q$ of $E$, respectively. Moreover, let $\mathfrak{M}_{E}$ indicate the family of all nonempty and bounded subsets of $E$ and $\mathfrak{N}_{E}$ indicate the family of all nonempty and relatively compact subsets of $E$.

Definition 1.0. A mapping $\mu: \mathfrak{M}_{E} \longrightarrow \mathbb{R}_{+}$is said to be a measure of noncompactness in $E$ if it satisfies the following conditions:

$1^{\circ}$ The family ker $\mu=\left\{Q \in \mathfrak{M}_{E}: \mu(Q)=0\right\}$ is nonempty and ker $\mu \subseteq \mathfrak{N}_{E}$.

$2^{\circ} Q_{1} \subset Q_{2} \Longrightarrow \mu\left(Q_{1}\right) \leq \mu\left(Q_{2}\right)$.

$3^{\circ} \mu(\bar{Q})=\mu(Q)$.

$4^{\circ} \mu(\operatorname{Conv} Q)=\mu(Q)$.

$5^{\circ} \mu\left(\lambda Q_{1}+(1-\lambda) Q_{2}\right) \leq \lambda \mu\left(Q_{1}\right)+(1-\lambda) \mu\left(Q_{2}\right)$ for $\lambda \in[0,1]$.

$6^{\circ}$ If $\left\{Q_{n}\right\}$ is a sequence of closed sets from $\mathfrak{M}_{E}$ such that $Q_{n+1} \subset Q_{n}$ for $n=1,2, \cdots$, and if $\lim _{n \rightarrow \infty} \mu\left(Q_{n}\right)=0$, then $Q_{\infty}=\cap_{n=1}^{\infty} Q_{n} \neq \varnothing$.

We say that a measure of noncompactness is regular [10] provided it satisfies additionally the following conditions:

$$
\begin{aligned}
& 7^{\circ} \mu\left(Q_{1} \cup Q_{2}\right)=\max \left\{\mu\left(Q_{1}\right), \mu\left(Q_{2}\right)\right\} . \\
& 8^{\circ} \mu\left(Q_{1}+Q_{2}\right) \leq \mu\left(Q_{1}\right)+\mu\left(Q_{2}\right) . \\
& 9^{\circ} \mu(\lambda Q)=|\lambda| \mu(Q) \text { for } \lambda \in \mathbb{R} . \\
& 10^{\circ} \text { ker } \mu=\mathfrak{N}_{E} .
\end{aligned}
$$


For example, $\alpha$ and $\chi$ are regular measures of noncompactness on $Q$.

Definition 1.1. [7] Let $E_{1}$ and $E_{2}$ be two Banach spaces and $\mu_{1}$ and $\mu_{2}$ be arbitrary measures of noncompactness on $E_{1}$ and $E_{2}$ respectively. An operator $T$ from $E_{1}$ to $E_{2}$ is called a $\left(\mu_{1}, \mu_{2}\right)$-condensing operator if it is continuous and for every bounded noncompact set $\Omega \subset E_{1}$ such that for $\Omega \notin \operatorname{ker} \mu_{1}$, the following inequality holds

$$
\mu_{2}(T(\Omega))<\mu_{1}(\Omega) .
$$

The contractive maps and the compact maps are condensing if we take as measures of noncompactness the diameter of a set and the indicator function of a family of non-relatively compact sets, respectively [7]. In 1955, Darbo published a fixed point theorem [16], using the concept of measures of noncompactness, which guarantees the existence of fixed point for condensing operators. Darbo's theorem has provided an abundance of applications in the existence of solutions for differential and integral equations (c.f. $[2,3,4,9,12,13,14,17,18,27,28,29]$ ). It extends both the classical Schauder's fixed point theorem and the Banach's contraction principle.

In 1969, Meir and Keeler [26] proved the following very interesting fixedpoint theorem, which is a generalization of the Banach contraction principle [8].

Definition 1.2. [26] Let $(X, d)$ be a metric space. A mapping $T$ on $X$ is said to be a Meir-Keeler contraction (MKC, for short) if for any $\varepsilon>0$, there exists $\delta>0$ such that

$$
\varepsilon \leq d(x, y)<\varepsilon+\delta \Rightarrow d(T x, T y)<\varepsilon,
$$

for all $x, y \in X$.

Definition 1.3. [5] Let $C$ be a nonempty subset of a Banach space $E$ and $\mu$ an arbitrary measure of noncompactness on $E$. We say that an operator $T: C \longrightarrow C$ is a Meir-Keeler condensing operator if for any $\varepsilon>0$, there exists $\delta>0$ such that

$$
\varepsilon \leq \mu(X)<\varepsilon+\delta \Rightarrow \mu(T(X))<\varepsilon,
$$

for any bounded subset $X$ of $C$.

Lim [25] introduced the notion of $L$-functions and characterized Meir-Keeler contractions in metric spaces.

Definition 1.4. (Lim [25]) A function $\varphi$ from $\mathbb{R}_{+}$into itself is called an $L$-function if $\varphi(0)=0, \varphi(s)>0$ for $s \in(0,+\infty)$, and for every $s \in(0,+\infty)$ there exists $\delta>0$ such that $\varphi(t) \leq s$, for all $t \in[s, s+\delta]$.

Definition 1.5. (Lim [5]) We say that $\theta: \mathbb{R}_{+} \longrightarrow \mathbb{R}_{+}$is a strictly $L$-function if $\theta(0)=0, \theta(s)>0$ for $s \in(0,+\infty)$, and for every $s \in(0,+\infty)$ there exists $\delta>0$ such that $\theta(t)<s$, for all $t \in[s, s+\delta]$.

The following results are given in [5] which are very useful in our study.

Theorem 1.1. Let $C$ be a nonempty, bounded, closed and convex subset of a Banach space $E$ and $\mu$ be an arbitrary measure of noncompactness on $E$. 
If $T: C \longrightarrow C$ is a continuous and Meir-Keeler condensing operator, then $T$ has at least one fixed point and the set of all fixed points of $T$ in $C$ is compact.

Theorem 1.2. Let $C$ be a nonempty and bounded subset of a Banach space $E$, $\mu$ an arbitrary measure of noncompactness on $E$ and $T: C \longrightarrow C$ be a continuous operator. Then $T$ is a Meir-Keeler condensing operator if and only if there exists an $L$-function $\varphi$ such that

$$
\mu(T X)<\varphi(\mu(X))
$$

for all $X \in \mathfrak{M}_{E}$ with $\mu(X) \neq 0$.

Corollary 1.3. Let $C$ be a nonempty, bounded, closed and convex subset of a Banach space $E$ and let $T: C \longrightarrow C$ be a continuous operator such that

$$
\mu(T X)<\varphi(\mu(X))
$$

for each $X \subseteq C$, where $\mu$ is an arbitrary measure of noncompactness and $\varphi$ is an $L$-function. Then $T$ has at least one fixed point and the set of all fixed points of $T$ in $C$ is compact.

Theorem 1.4. Let $C$ be a nonempty, bounded, closed and convex subset of a Banach space $E$ and let $T: C \longrightarrow C$ be a continuous operator such that

$$
\mu(T X) \leq \theta(\mu(X))
$$

for each $X \subseteq C$, where $\mu$ is an arbitrary measure of noncompactness and $\theta$ is a strictly $L$-function. Then $T$ has at least one fixed point and the set of all fixed points of $T$ in $C$ is compact.

Corollary 1.5. Let $C$ be a nonempty, bounded, closed and convex subset of a Banach space $E$ and let $F: C \longrightarrow E$ be an operator such that

$$
\|F x-F y\| \leq \theta(\|x-y\|)
$$

where $\theta$ is a nondecreasing and right continuous strictly $L$-function. Let $G: C \longrightarrow$ $E$ be a compact and continuous operator. Define $T(x):=F(x)+G(x)$ and assume that $T(x) \in C$ for all $x \in C$. Then $T$ has a fixed point in $C$ and the set of all fixed points of $T$ in $C$ is compact.

Recently, Karakaya et. al. [23] proved a tripled fixed point theorem for a class of condensing operators in Banach spaces. In this paper we prove some tripled fixed point theorems for Meir-Keleer condensing operator in a Banach space using $L$-functions via measures of noncompactness. Furthermore, we apply our results to establish the existence of solutions for a system of functional integral equations of Volterra type. 


\section{Tripled fixed point results for trivariate Meir-Keeler condens- ing operators}

In this section we introduce the notion of a trivariate Meir-Keeler condensing operator and prove some tripled fixed point results.

Definition 2.1. [15] An element $(x, y, z) \in X \times X$ is called a tripled fixed point of the operator $F: X \times X \times X \longrightarrow X$ if $F(x, y, z)=x, F(y, x, z)=y$ and $F(z, y, x)=$ $z$.

Theorem 2.1. [10] Suppose $\mu_{1}, \mu_{2}, \cdots, \mu_{n}$ are measures of noncompactness on Banach spaces $E_{1}, E_{2}, \cdots, E_{n}$, respectively. Moreover assume that the function $F: \mathbb{R}_{+}^{n} \longrightarrow \mathbb{R}_{+}$is convex and $F\left(x_{1}, \cdots, x_{n}\right)=0$ if and only if $x_{i}=0$ for $i=1,2, \cdots, n$. Then

$$
\mu(X)=F\left(\mu_{1}\left(X_{1}\right), \mu_{2}\left(X_{2}\right), \cdots, \mu_{n}\left(X_{n}\right)\right)
$$

defines a measure of noncompactness on $E_{1} \times E_{2} \times \cdots \times E_{n}$ where $X_{i}$ denotes the natural projections of $X$ into $E_{i}$ for $i=1,2, \cdots, n$.

Similar to [6] we can construct the following example.

Example 2.1. Let $\mu$ be a measure of noncompactness on a Banach space $E$. If we consider $F_{1}(x, y, z)=\max \{x, y, z\}$ and $F_{2}(x, y, z)=x+y+z$ for $x, y, z \in \mathbb{R}_{+}^{3}$, then conditions of Theorem 2.1 are satisfied. Therefore, $\tilde{\mu}_{1}(X):=\max \left\{\mu\left(X_{1}\right), \mu\left(X_{2}\right)\right.$, $\left.\mu\left(X_{3}\right)\right\}$ and $\tilde{\mu}_{2}(X):=\mu\left(X_{1}\right)+\mu\left(X_{2}\right)+\mu\left(X_{3}\right)$ define measures of noncompactness in the space $E \times E \times E$ where $X_{i}$ denotes the natural projections of $X$ into $E_{i}$ for $i=1,2,3$.

Now, we define the notion of a trivariate Meir-Keeler condensing operator and use to prove our first result.

Definition 2.2. Let $C$ be a nonempty subset of a Banach space $E$ and $\mu$ an arbitrary measure of noncompactness on $E$. We say that $T: C \times C \times C \longrightarrow C$ is a MeirKeeler condensing operator if for any $\varepsilon>0$, there exists $\delta>0$ such that

$$
\varepsilon \leq \max \left\{\mu\left(X_{1}\right), \mu\left(X_{2}\right), \mu\left(X_{3}\right)\right\}<\varepsilon+\delta \Longrightarrow \mu\left(T\left(X_{1} \times X_{2} \times X_{3}\right)\right)<\varepsilon
$$

for any bounded subsets $X_{1}, X_{2}, X_{3}$ of $C$.

Theorem 2.2. Let $C$ be a nonempty, bounded closed and convex subset of a Banach space $E$ and $\mu$ an arbitrary measure of noncompactness on $E$. If $T: C \times C \times C \longrightarrow C$ is a continuous Meir-Keeler condensing operator then $T$ has at least one tripled fixed point.

Proof. From Example 2.1, we note that $\tilde{\mu}_{1}(X):=\max \left\{\mu\left(X_{1}\right), \mu\left(X_{2}\right), \mu\left(X_{3}\right)\right\}$ for any bounded subset $X \subset E \times E \times E$ defines a measure of noncompactness on $E \times E \times E$ where $X_{i}$ denotes the natural projections of $X$ into $E_{i}$ for $i=1,2,3$. Also the operator $\mathrm{G}: \mathrm{C} \times \mathrm{C} \times \mathrm{C} \longrightarrow \mathrm{C} \times \mathrm{C} \times \mathrm{C}$ given by

$$
G(x, y, z):=(T(x, y, z), T(y, x, z), T(z, y, x))
$$


is clearly continuous on $C \times C \times C$. Now we claim that $G$ satisfies all the conditions of Theorem 1.1. To prove this, let $\varepsilon>0$ and $\delta(\varepsilon)>0$ be as in Definition 2.2. If $X$ is a bounded subset of $C \times C \times C$ such that

$$
\varepsilon \leq \tilde{\mu}(X)<\varepsilon+\delta
$$

then

$$
\varepsilon \leq \max \left\{\mu\left(X_{1}\right), \mu\left(X_{2}\right), \mu\left(X_{3}\right)\right\}<\varepsilon+\delta .
$$

By $2^{\circ}$ of Definition 1.0 and (2.1), we get

$$
\begin{aligned}
\tilde{\mu}(G(X)) & \\
& \leq \tilde{\mu}\left(T\left(X_{1} \times X_{2} \times X_{3}\right) \times T\left(X_{2} \times X_{1} \times X_{3}\right) \times T\left(X_{3} \times X_{2} \times X_{1}\right)\right\}<\varepsilon+\delta \\
& =\max \left\{\mu\left(T\left(X_{1} \times X_{2} \times X_{3}\right)\right), \mu\left(T\left(X_{2} \times X_{1} \times X_{3}\right)\right), \mu\left(T\left(X_{3} \times X_{2} \times X_{1}\right)\right)\right\} \\
& <\varepsilon .
\end{aligned}
$$

Hence, from Theorem 1.1, $G$ has at least one fixed point in $C \times C \times C$. Now the conclusion of theorem follows from the fact that every fixed point of $G$ is a tripled fixed point of $T$.

This completes the proof of the theorem.

Now, we prove a tripled fixed point theorem by using $L$-functions.

Theorem 2.3. Let $C$ be a nonempty, bounded closed and convex subset of a Banach space $E$ and $\varphi$ an $L$-function. Suppose that for any measure of noncompactness $\mu$ on $E$, the continuous operator $T: C \times C \times C \longrightarrow C$ satisfies

$$
\mu\left(T\left(X_{1} \times X_{2} \times X_{3}\right)\right)<\frac{1}{3} \varphi\left(\mu\left(X_{1}\right)+\mu\left(X_{2}\right)+\mu\left(X_{3}\right)\right),
$$

for any subsets $X_{1}, X_{2}, X_{3}$ of $C$. Then $G$ has at least one tripled fixed point.

Proof. Similarly to the proof of Theorem 2.2, we define a mapping $G: C \times C \times$ $\mathrm{C} \longrightarrow \mathrm{C} \times \mathrm{C} \times \mathrm{C}$ by

$$
G(x, y, z):=(T(x, y, z), T(y, x, z), T(z, y, x))
$$

which is continuous. On the other hand, from Example 2.1, we have $\tilde{\mu}(X):=$ $\mu\left(X_{1}\right)+\mu\left(X_{2}\right)+\mu\left(X_{3}\right)$ which defines a measure of noncompactness on $E \times E \times E$ where $X_{1}, X_{2}, X_{3}$ denote the natural projections of $X$. Now let $X \subset C \times C \times C$ be any nonempty subset. Then by $2^{\circ}$ of Definition 1.0 and (2.2) we obtain

$$
\begin{aligned}
\tilde{\mu}(G(X)) & \\
& \leq \tilde{\mu}\left(T\left(X_{1} \times X_{2} \times X_{3}\right) \times T\left(X_{2} \times X_{1} \times X_{3}\right) \times T\left(X_{3} \times X_{2} \times X_{1}\right)\right\}<\varepsilon+\delta \\
& =\mu\left(T\left(X_{1} \times X_{2} \times X_{3}\right)\right)+\mu\left(T\left(X_{2} \times X_{1} \times X_{3}\right)\right)+\mu\left(T\left(X_{3} \times X_{2} \times X_{1}\right)\right) \\
& <\varphi\left(\mu\left(X_{1}\right)+\mu\left(X_{2}\right)+\mu\left(X_{3}\right)\right) \\
& \leq \varphi(\tilde{\mu}(X)) .
\end{aligned}
$$

Therefore, all the conditions of Corollary 1.3 are satisfied. Hence $G$ has a fixed point or equivalently $T$ has a tripled fixed point. 
This completes the proof of the theorem.

Our next result is a consequence of Theorem 1.4.

Theorem 2.4. Let $C$ be a nonempty, bounded closed and convex subset of a Banach space $E$ and $\theta$ a strictly $L$-function. Suppose that for any measure of noncompactness $\mu$ on $E$, the continuous operator $T: C \times C \times C \longrightarrow C$ satisfies

$$
\mu\left(T\left(X_{1} \times X_{2} \times X_{3}\right)\right) \leq \frac{1}{3} \theta\left(\mu\left(X_{1}\right)+\mu\left(X_{2}\right)+\mu\left(X_{3}\right)\right),
$$

for any subsets $X_{1}, X_{2}, X_{3}$ of $C$. Then $G$ has at least one tripled fixed point. Proof. Its proof is similar to the proof of Theorem 2.2.

Corollary 2.5. Let $C$ be a nonempty, bounded, closed and convex subset of a Banach space $E$ and let $F: C \times C \times C \longrightarrow E$ be an operator such that

$$
\|F(x, y, z)-F(u, v, w)\| \leq \frac{1}{3} \theta(\|x-u\|+\|y-v\|+\|z-w\|)
$$

where $\theta$ is a nondecreasing and upper semicontinuous strictly $L$-function. Assume that $G: C \times C \times C \longrightarrow E$ is a compact, continuous operator. Define $T(x, y, z):=F(x, y, z)+G(x, y, z)$ and assume that $T(x, y, z) \in C$ for all $x, y . z \in C$. Then $F$ has at least a tripled fixed point.

Proof. Let $\mu: \mathfrak{M}_{E} \rightarrow \mathbb{R}_{+}$be Kuratowski measure of noncompactness. Moreover, let $X_{1}, X_{2}, X_{3}$ be nonempty subsets of $C$. Since $\theta$ is nondecreasing, by (2.4) we have

$$
\begin{aligned}
\|F(x, y, z)-F(u, v, w)\| & \leq \frac{1}{3} \theta(\|x-u\|+\|y-v\|+\|z-w\|) \\
& \leq \frac{1}{3} \theta(\operatorname{diam}\|x-u\|+\operatorname{diam}\|y-v\|+\operatorname{diam}\|z-w\|)
\end{aligned}
$$

and

$$
\operatorname{diam}\left(F\left(X_{1} \times X_{2} \times X_{3}\right)\right) \leq \frac{1}{3} \theta\left(\operatorname{diam}\left(X_{1}\right)+\operatorname{diam}\left(X_{2}\right)+\operatorname{diam}\left(X_{3}\right)\right) .
$$

Since $\theta$ is right continuous, similarly to the proof of Corollary 2.5 , we have

$$
\mu\left(F\left(X_{1} \times X_{2} \times X_{3}\right)\right) \leq \frac{1}{3} \theta\left(\mu\left(X_{1}\right)+\mu\left(X_{2}\right)+\mu\left(X_{3}\right)\right)
$$

and since $G$ is compact

$$
\begin{aligned}
\mu\left(T\left(X_{1} \times X_{2} \times X_{3}\right)\right) & =\mu\left((F+G)\left(X_{1} \times X_{2} \times X_{3}\right)\right) \\
& \leq \mu\left(F\left(X_{1} \times X_{2} \times X_{3}\right)+G\left(X_{1} \times X_{2} \times X_{3}\right)\right) \\
& \leq \mu\left(F\left(X_{1} \times X_{2} \times X_{3}\right)\right)+\mu\left(G\left(X_{1} \times X_{2} \times X_{3}\right)\right) \\
& \leq \frac{1}{3} \theta\left(\mu\left(X_{1}\right)+\mu\left(X_{2}\right)+\mu\left(X_{3}\right)\right) .
\end{aligned}
$$

Now, by applying Theorem 2.4, we get the desired result. 
This completes the proof of the theorem.

\section{Applications}

In this section, we apply our results to prove the existence of solutions for a system of functional integral equations of Volterra type.

Let $B C\left(\mathbb{R}_{+}\right)$be the Banach space of all bounded and continuous functions on $\mathbb{R}_{+}$ equipped with the standard norm

$$
\|x\|_{\infty}=\sup \{|x(t)|: t \geq 0\} .
$$

For any nonempty bounded subset $X$ of $B C\left(\mathbb{R}_{+}\right), x \in X, A>0$ and $\varepsilon>0$, let

$$
\begin{aligned}
& \omega^{A}(x, \varepsilon)=\sup \{|x(t)-x(u)|: t, u \in[0, A],|t-u| \leq \varepsilon\} \\
& \omega^{A}(X, \varepsilon)=\sup \left\{\omega^{A}(x, \varepsilon): x \in X\right\}, \\
& \omega_{0}^{A}(X)=\lim _{\varepsilon \rightarrow 0} \omega^{A}(X, \varepsilon), \\
& \omega_{0}(X)=\lim _{A \rightarrow \infty} \omega_{0}^{A}(X), \\
& X(t)=\{x(t): x \in X\}
\end{aligned}
$$

and

$$
\mu(X)=\frac{1}{2}\left(\omega_{0}(X)+\limsup _{t \rightarrow \infty} \operatorname{diam} X(t)\right) .
$$

The function $\mu$ is a measure of noncompactness in the space $B C\left(\mathbb{R}_{+}\right)$(in the sense of Definition 1.1) (cf. [10], [13]).

Theorem 3.1. Assume that the following conditions are satisfied:

(i) $f: \mathbb{R}_{+} \times \mathbb{R} \times \mathbb{R} \times \mathbb{R} \times \mathbb{R} \longrightarrow \mathbb{R}$ is continuous and there exist nondecreasing and upper semicontinuous strictly $L$-function $\theta$ such that

$$
\begin{aligned}
\left|f\left(t, x_{1}, x_{2}, x_{3}, x_{4}\right)-f\left(t, y_{1}, y_{2}, y_{3}, y_{4}\right)\right| & \leq \\
& \frac{1}{2}\left(\theta\left(\left|x_{1}-y_{1}\right|+\left|x_{2}-y_{2}\right|+\left|x_{3}-y_{3}\right|\right)\right)+\left|x_{4}-y_{4}\right|,
\end{aligned}
$$

(ii) $M:=\sup \left\{|f(t, 0,0,0,0)|: t \in \mathbb{R}_{+}\right\}<\infty$.

(iii) $g: \mathbb{R}_{+} \times \mathbb{R}_{+} \times \mathbb{R} \times \mathbb{R} \times \mathbb{R} \longrightarrow \mathbb{R}$ is continuous and there exists a positive constant $D$ such that

$$
D=\sup \left\{\left|\int_{0}^{t} g(t, s, x(s), y(s), z(s)) d s\right|: t \in \mathbb{R}_{+}, x, y, z \in B C\left(\mathbb{R}_{+}\right)\right\} .
$$

Moreover,

$$
\lim _{t \rightarrow \infty}\left|\int_{0}^{t}[g(t, s, x(s), y(s), z(s))-g(t, s, u(s), v(s), w(s)) d s]\right|=0
$$

uniformly with respect to $x, y, z, u, v, w \in B C\left(\mathbb{R}_{+}\right)$.

(iv) There exists a positive solution $r_{0}$ of the inequality

$$
\frac{1}{3} \theta(3 r)+M+D \leq r .
$$


Then the system of functional integral equations

$$
\left\{\begin{array}{l}
x(t)=f(t, x(t), y(t), z(t))+\int_{0}^{t} g(t, s, x(s), y(s), z(s)) d s \\
y(t)=f(t, y(t), x(t), z(t))+\int_{0}^{t} g(t, s, y(s), x(s), z(s)) d s \\
z(t)=f(t, z(t), y(t), x(t))+\int_{0}^{t} g(t, s, z(s), y(s), x(s)) d s
\end{array}\right.
$$

has at least one solution in the space $B C\left(\mathbb{R}_{+}\right) \times B C\left(\mathbb{R}_{+}\right) \times B C\left(\mathbb{R}_{+}\right)$.

For $x, y, z \in B C\left(\mathbb{R}_{+}\right)$, let

$$
\|(x, y, z)\|_{B C\left(\mathbb{R}_{+}\right)^{3}}=\|x\|_{\infty}+\|y\|_{\infty}+\|z\|_{\infty} .
$$

We can easily prove that the solution of (3.5) in $B C\left(\mathbb{R}_{+}\right) \times B C\left(\mathbb{R}_{+}\right) \times B C\left(\mathbb{R}_{+}\right)$is equivalent to the tripled fixed point of $G$.

The proof depends upon the following lemma.

Lemma 3.2. Assume that $g$ satisfies the hypothesis (iii) of Theorem 3.1. Then $G: B C\left(\mathbb{R}_{+}\right) \times B C\left(\mathbb{R}_{+}\right) \times B C\left(\mathbb{R}_{+}\right) \longrightarrow B C\left(\mathbb{R}_{+}\right)$defined by

$$
G(x, y, z)(t)=\int_{0}^{t} g(t, s, x(s), y(s), z(s)) d s
$$

is a compact and continuous operator.

Proof. First we show that $G(x, y, z)(t)$ is continuous for any $x, y, z \in B C\left(\mathbb{R}_{+}\right)$. Let $x, y, z \in B C\left(\mathbb{R}_{+}\right)$and $\varepsilon>0$. Take $u, v, w \in B C\left(\mathbb{R}_{+}\right)$with $\|(x, y, z)-$ $(u, v, w) \|_{B C\left(\mathbb{R}_{+}\right)^{3}<\varepsilon \text {. Then, by condition (ii) and (3.2), there exists } T>0 \text { such }}$ that for $t>T$, we have

$$
\begin{aligned}
& |G(x, y, z)(t)-G(u, v, w)(t)| \leq \\
& \quad \int_{0}^{t}|g(t, s, x(s), y(s), z(s)) d s-g(t, s, u(s), v(s), w(s)) d s| \leq \varepsilon,
\end{aligned}
$$

for any $x, y, z \in B C\left(\mathbb{R}_{+}\right)$. Also if $t \in[0, T]$, then the first inequality in (3.7) implies that

$$
|G(x, y, z)(t)-G(u, v, w)(t)| \leq T \vartheta_{T}(\varepsilon)
$$

where

$$
\begin{aligned}
\vartheta_{T}(\varepsilon)= & \sup \{|g(t, s, x . y . z)-g(t, s, u, v, w)|: t \in[0, T], x, y, z, u, v, w \in[-b, b], \\
\| & (x, y, z)-(u, v, w) \|_{\left.B C\left(\mathbb{R}_{+}\right)^{3}<\varepsilon\right\},}
\end{aligned}
$$

with $b=\|x\|_{\infty}+\|y\|_{\infty}+\|z\|_{\infty}+\varepsilon$. By using the continuity of $g$ on $[0, T] \times[0, T] \times$ $[-b, b] \times[-b, b] \times[-b, b]$, we have $\vartheta_{T}(\varepsilon) \rightarrow 0$ as $\varepsilon \rightarrow 0$. Thus, $G$ is a continuous function on $B C\left(\mathbb{R}_{+}\right) \times B C\left(\mathbb{R}_{+}\right) \times B C\left(\mathbb{R}_{+}\right)$. Now, let $X_{1}, X_{2}, X_{3}$ be nonempty and bounded subsets of $B C\left(\mathbb{R}_{+}\right)$, and assume that $T>0$ and $\varepsilon>0$ are arbitrary constants. Let $t_{1}, t_{2} \in[0, T]$ with $\left|t_{1}-t_{2}\right| \leq \varepsilon$ and $(x, y, z) \in X_{1} \times X_{2} \times X_{3}$. We have $\left|G(x, y, z)\left(t_{1}\right)-G(x, y, z)\left(t_{2}\right)\right| \leq$

$\left|\int_{0}^{t_{1}} g\left(t_{1}, s, x(s), y(s), z(s)\right) d s-\int_{0}^{t_{2}} g\left(t_{2}, s, x(s), y(s), z(s)\right) d s\right| \leq T \omega_{r}^{T}(g, \varepsilon)+U_{r}^{T} \varepsilon$ 
where $r=\sup _{x, y, z \in X}\left\{\|x\|_{\infty}+\|y\|_{\infty}+\|z\|_{\infty}\right\}$,

$$
\begin{gathered}
\omega_{r}^{T}(g, \varepsilon)=\sup \left\{\left|g\left(t_{1}, s, x, y, z\right)-g\left(t_{2}, s, x, y, z\right)\right|:\right. \\
\left.t_{1}, t_{2} \in[0, T], x, y, z \in[-r, r],\left|t_{1}-t_{2}\right| \leq \varepsilon\right\}, \\
U_{r}^{T}=\sup \{|g(t, s, x, y, z)|: t \in[0, T], x, y, z \in[-r, r]\} .
\end{gathered}
$$

Since $(x, y, z)$ was arbitrary, we obtain

$$
\omega^{T}\left(G\left(X_{1} \times X_{2} \times X_{3}\right), \varepsilon\right) \leq T \omega_{r}^{T}(g, \varepsilon)+U_{r}^{T} \varepsilon .
$$

On the other hand, by the uniform continuity of $g$ on $[0, T] \times[0, T] \times[-r, r] \times$ $[-r, r] \times[-r, r]$, we have $\omega_{r}^{T}(g, \varepsilon) \rightarrow 0$ as $\varepsilon \rightarrow 0$. Therefore we obtain $\omega_{0}^{T}\left(G\left(X_{1} \times X_{2} \times X_{3}\right)\right)=0$ and, finally

$$
\omega_{0}\left(G\left(X_{1} \times X_{2} \times X_{3}\right)\right)=0 .
$$

In addition, for arbitrary $(x, y, z),(u, v, w) \in X_{1} \times X_{2} \times X_{3}$ and $t \in \mathbb{R}_{+}$, we have

$$
\begin{aligned}
& |G(x, y, z)(t)-G(u, v, w)(t)| \leq \int_{0}^{t} \mid \\
& \quad g(t, s, x(s), y(s), z(s)) d s-g(t, s, u(s), v(s), w(s)) d s \mid \leq \beta(t)
\end{aligned}
$$

where

$$
\begin{array}{r}
\beta(t)=\sup \{|g(t, s, x(s), y(s), z(s))-g(t, s, u(s), v(s), w(s))|: \\
\left.t, s \in[0, T] ; x, y, z, u, v, w \in B C\left(\mathbb{R}_{+}\right)\right\} .
\end{array}
$$

Thus, we have

$$
\operatorname{diam} G\left(X_{1} \times X_{2} \times X_{3}\right)(t) \leq \beta(t)
$$

Taking the limit as $t \rightarrow \infty$ in the inequality (3.11) and using (iii) we get

$$
\limsup _{t \rightarrow \infty} \operatorname{diam} G\left(X_{1} \times X_{2} \times X_{3}\right)(t)=0
$$

Further, combining (3.10) and (3.12), we get

$$
\limsup _{t \rightarrow \infty} \operatorname{diam} G\left(X_{1} \times X_{2} \times X_{3}\right)(t)+\omega_{0}\left(G\left(X_{1} \times X_{2} \times X_{3}\right)\right)=0
$$

or equivalently

$$
\mu\left(G\left(X_{1} \times X_{2} \times X_{3}\right)\right)=0 .
$$

Thus, $G$ is compact and the proof is complete.

Proof of Theorem 3.1. We define the operators $F, T: B C\left(\mathbb{R}_{+}\right) \times B C\left(\mathbb{R}_{+}\right) \times B C\left(\mathbb{R}_{+}\right) \rightarrow$ $B C\left(\mathbb{R}_{+}\right) \times B C\left(\mathbb{R}_{+}\right) \times B C\left(\mathbb{R}_{+}\right)$by

$$
F(x, y, z)(t)=f(t, x(t), y(t), z(t))
$$

and

$$
T(x, y, z)(t)=f(t, x(t), y(t), z(t))+\int_{0}^{t} g(t, s, x(s), y(s), z(s)) d s
$$


Using conditions (i)-(iv), for arbitrarily fixed $t \in \mathbb{R}_{+}$, we have

$$
\begin{aligned}
& G(x, y, z)(t) \\
& \leq\left|f(t, x(t), y(t), z(t))+\int_{0}^{t} g(t, s, x(s), y(s), z(s)) d s-f(t, 0,0,0,0)\right| \\
& \quad+|f(t, 0,0,0,0)| \\
& \leq \frac{1}{3} \theta(|x(t)|+|y(t)|+|z(t)|)+\left|\int_{0}^{t} g(t, s, x(s), y(s), z(s)) d s\right|+|f(t, 0,0,0,0)| \\
& \leq \frac{1}{3} \theta(|x(t)|+|y(t)|+|z(t)|)+M+D
\end{aligned}
$$

Since by assumption (ii) the function $\theta$ is nondecreasing, we get

$$
\|G(x, y, z)\| \leq \frac{1}{3} \theta\left(\|x\|_{\infty}+\|y\|_{\infty}+\|z\|_{\infty}\right)+M+D
$$

Thus, keeping in mind assumption (iv) we infer that $T$ is a self mapping of the ball $\bar{B}_{r_{0}}$. Next, by condition (ii) of Theorem 3.1, it is obvious that $F$ and $G$ for $x, y, z \in B C\left(\mathbb{R}_{+}\right)$are continuous functions, and

$$
\|F(x, y, z)-F(u, v, w)\|<\theta\left(\|(x, y, z)-(u, v, w)\|_{\left.B C\left(\mathbb{R}_{+}\right)^{3}\right) .}\right.
$$

Let $\mu: \mathfrak{M}_{E} \rightarrow \mathbb{R}_{+}$be the Kuratowski measure of noncompactness defined by (1.1). Using Theorem 2.4, we get

$$
\mu(F(x)) \leq \theta(\mu(x))
$$

Thus, $F$ is a Meir-Keeler condensing operator. Finally, since $T(x, y, z)=F(x, y, z)+$ $G(x, y, z), G$ is a compact and continuous operator and $F$ is a continuous MeirKeeler condensing operator, by Corollary 2.5, $T$ has a fixed point.

\section{References}

[1] A. Aghajani, R. Allahyari, M. Mursaleen, A generalization of Darbo's theorem with application to the solvability of systems of integral equations, Journal of Computational and Applied Mathematics 260 (2014) 68-77.

[2] A. Aghajani, J. Banaś, Y. Jalilian, Existence of solution for a class nonlinear Volterra singular integral, Computer and Mathematics with Applications 62 (2011 ) 1215-1227

[3] A. Aghajani, Y. Jalilian, Existence and global attractivity of solutions of a nonlinear functional integral equation, Commun Nonlinear Sci. Numer. Simulat. 15 (2010) 3306-3312.

[4] A. Aghajani, Y. Jalilian, Existence of nondecreasing positive solution for a system of singular integral equations, Mediter. J. Math., 8 (2011) 563-586. 
[5] A. Aghajani , M. Mursaleen, A. Shole Haghighi, Fixed point theorems for Meir-Keeler condensing operators via measure of noncompactness, Acta Math. Sci. (To appear).

[6] A. Aghajani, N. Sabzali, Existence of coupled fixed points via measure of noncompactness and applications, Journal of Nonlinear and Convex Analysis 15 (2014) 941-952.

[7] R.R. Akmerov, M.I. Kamenski, A.S. Potapov, A.E. Rodkina, B.N. Sadovskii, Measures of Noncompactness and Condensing Operators, Birkhäuser Verlag, Basel, 1992.

[8] S. Banach, Sur les opérations dans les ensembles abstraits et leur application aux équations intégrales, Fund. Math. 3 (1922) 133-181.

[9] J. Banaś, B. C. Dhage, Global asymptotic stability of solutions of a functional integral equation, Nonlinear Anal. 69 (2008) 1945-1952

[10] J. Banaś, K. Goebel, Measures of Noncompactness in Banach Spaces, Lecture Notes in Pure and Applied Mathematics, Vol. 60, Dekker, New York, 1980.

[11] J. Banaś, M. Mursaleen, Sequence Spaces and Measures of Noncompactness With Applications to Differential and Integral Equations, Springer, New Delhi, 2014.

[12] J. Banaś, D. O'Regan , K. Sadarangani, On solutions of a a quadratic hammerstein integral equation on an unbounded interval, Dynamic Systems and Applications 18 (2009) 251-264.

[13] J. Banaś, R. Rzepka, an application of a measure of noncompactness in the study of asymptotic stability, Appl. Math. Lett. 16 (2003) 1-6.

[14] J. Banaś, K. Sadarangani, Compactness conditions in the study of functional, differential, and integral equations, Volume 2013 (2013), Article ID 819315, 14 pages, doi: 10.1155/2013/819315.

[15] S. S. Chang, Y. J. Cho, N. J. Huang, Coupled fixed point theorems with applications, J. Korean Math. Soc. 33 (1996) No. 3, pp. 575-585.

[16] G. Darbo, Punti uniti in trasformazioni a codominio non compatto, Rend. Sem. Mat. Univ. Padova, 24 (1955) 84-92.

[17] M. A. Darwish, J. Henderson, D. O'Regan, Existence and asymptotic stability of solutions of a perturbed fractional functional-integral equation with linear modification of the argument, Bull. Korean Math. Soc. 48 (2011) 539-553.

[18] S. Djebali, D. O'Regan, Z. Sahnoun, On the solvability of some operator equations and inclusions in banach spaces with the weak topology, Applied Analysis 15 (2011) 125-140.

[19] J. Garcia-Falset, Existence of fixed points and measures of weak noncompactness, Nonlinear Anal. 71 (2009) 2625-2633. 
[20] L.S. Goldenštein, I.T. Gohberg, A.S. Markus, Investigations of some properties of bounded linear operators with their q-norms, Učen. Zap. Kishinevsk. Univ., 29 (1957) 29-36.

[21] L.S. Goldenštein, A.S. Markus, On a measure of noncompactness of bounded sets and linear operators, in: Studies in Algebra and Mathematical Analysis, Kishinev, 1965, pp. 45-54.

[22] V. Istrăţescu, On a measure of noncompactness, Bull. Math. Soc. Sci. Math. R.S. Roumanie (N.S), 16 (1972) 195-197.

[23] V. Karakaya, N. H. Bouzara, K. Dogan, Y. Atalan, Existence of tripled fixed points for a class of condensing operators in Banach spaces, The Scientific World Journal, Volume 2014 (2014), Article ID 541862, 9 pages.

[24] K. Kuratowski, Sur les espaces complets. Fund. Math.15 (1930) 301-309.

[25] T.C. Lim, On characterizations of Meir-Keeler contractive maps, Nonlinear Anal. 46 (2001) 113-120.

[26] A. Meir, E. Keeler, A theorem on contraction mappings, J. Math. Anal. Appl. 28 (1969) 326-329.

[27] M. Mursaleen, A. Alotaibi, Infinite system of differential equations in some BK spaces, Abstract Appl. Anal., Volume 2012 (2012), Article ID 863483, 20 pages, doi:10.1155/2012/863483.

[28] M. Mursaleen, S. A. Mohiuddine, Applications of measures of noncompactness to the infinite system of differential equations in $\ell_{p}$ spaces, Nonlinear Analysis (TMA), 75 (2012) 2111-2115.

[29] M. Mursaleen, Application of measure of noncompactness to infinite system of differential equations, Canad. Math. Bull., 56 (2013) 388-394.

[30] M. Mursaleen, A. K. Noman, Hausdorff measure of noncompactness of certain matrix operators on the sequence spaces of generalized means, Jour. Math. Anal. Appl., 417 (2014) 96-111.

Department of Mathematics, Faculty of Science,

King Abdulaziz University,

P.O. Box 80203, Jeddah 21589, Saudi Arabia

emails : mathker11@hotmail.com; mohiuddine@gmail.com

Department of Mathematics,

Aligarh Muslim University,

Aligarh 202002, India

Email: mursaleenm@gmail.com 